\title{
Clinical EFT as an Evidence-Based Practice for the Treatment of Psychological and Physiological Conditions
}

\author{
Dawson Church \\ National Institute for Integrative Healthcare, Fulton, USA \\ Email: dawsonchurch@gmail.com
}

Received May $24^{\text {th }}$, 2013; revised July $5^{\text {th }}$, 2013; accepted July $15^{\text {th }}$, 2013

\begin{abstract}
Copyright (C) 2013 Dawson Church. This is an open access article distributed under the Creative Commons Attribution License, which permits unrestricted use, distribution, and reproduction in any medium, provided the original work is properly cited.
\end{abstract}

\begin{abstract}
Emotional Freedom Techniques (EFT) has moved in the past two decades from a fringe therapy to widespread professional acceptance. This paper defines Clinical EFT, the method validated in many research studies, and shows it to be an "evidence-based" practice. It describes standards by which therapies may be evaluated, such as those of the American Psychological Association (APA) Division 12 Task Force, and reviews the studies showing that Clinical EFT meets these criteria. Several research domains are discussed, summarizing studies of: 1) psychological conditions such as anxiety, depression, phobias, and posttraumatic stress disorder (PTSD); 2) physiological problems such as pain and autoimmune conditions; 3) professional and sports performance; and 4) the physiological mechanisms of action of Clinical EFT. The paper lists the conclusions that may be drawn from this body of evidence, which includes 23 randomized controlled trials and 17 within-subjects studies. The three essential ingredients of Clinical EFT are described: exposure, cognitive shift, and acupressure. The latter is shown to be an essential ingredient in EFT's efficacy, and not merely a placebo. New evidence from emerging fields such as epigenetics, neural plasticity, psychoneuroimmunology, and evolutionary biology confirms the central link between emotion and physiology, and points to somatic stimulation as the element common to emerging psychotherapeutic methods. The paper outlines the next steps in EFT research, such as smartphone-based data gathering, large-scale group therapy, and the use of biomarkers. It concludes that Clinical EFT is a stable and mature method with an extensive evidence base. These characteristics have led to growing acceptance in primary care settings as a safe, rapid, reliable, and effective treatment for both psychological and medical diagnoses.
\end{abstract}

Keywords: Research; Evidence-Based; Emotional Freedom Techniques; EFT; Exposure; Cognitive Therapy; Acupressure; Placebo

\section{Introduction}

Emotional Freedom Techniques (EFT; Craig, 2010) has moved in the past two decades from a novel intervention derived from Thought Field Therapy (TFT; Callahan, 2001) to an "evidence-based" practice in its own right. Evidence-based practices are methods that meet formally established criteria for efficacy (Melnyk \& Fineout-Overholt, 2005; Beautler, Norcross, \& Beutler, 2005). There are several organizations that define and publish such standards. One of these is the US government's Food and Drug Administration (FDA; Food and Drug Administration, 1998). Another is the UK government's National Institute for Clinical Excellence (NICE; National Institute for Clinical Excellence, 2009). The most influential set of standards in the field of psychology is the one published by the Task Force on Empirically Validated Treatments set up by Division 12 (Clinical Psychology) of the American Psychological Association (APA; Chambless et al., 1996, 1998; Chambless \& Hollon, 1998). For convenience these are referred to as "APA" standards.

\section{The Need for a Definition of Clinical EFT}

Millions of people worldwide are engaged with EFT. A Google Analytics search showed that in June 2013, 9,143,300 individuals searched for "tapping," "EFT," and related terms such as "EFT therapy” (Google Trends, 2013). Analysis of visitors to the five most-visited EFT web sites shows 6,965,000 unique visitors in that same month (Traffic Estimate, 2013). As of 2013 over 2 million individuals had downloaded from the Internet The EFT Manual (Craig \& Fowlie, 1995) or The EFT Mini-Manual (Church, 2013). Thousands of videos made by hundreds of different individuals appear on YouTube, social networking sites, and individual websites, attesting to the popularity of the method as well as practitioners' conviction of its efficacy.

This proliferation of sources offering EFT presents both challenges and opportunities. Many of these sources offer variants of EFT. A few sources present the original EFT method as detailed in the manual (Craig, 2010; Church, 2013a). Others present methods that share only a name with EFT while being 
devoid of an accurate description of any of its methods. The remainder are found somewhere on a spectrum between the two extremes. Tangentially, many variants of EFT have been developed by others, and there are even variants on the variants (Feinstein, 2009).

This crowded field of candidates has led to the question of what, exactly, is EFT? This paper seeks to answer that question. To facilitate review, this paper uses the term "Clinical EFT" defined as follows: "Clinical EFT is the 'evidence-based' method that has been validated in research studies that meet APA standards." These studies typically use a manual, The EFT Manual (Craig, 2010; Church, 2013a), which ensures that the method as tested in one study is the same method being tested in another study. The studies typically apply EFT with fidelity to the method described in the manual, and many studies describe methods of testing therapist fidelity to the method. Training of practitioners is expected to adhere to the method as described in the manual, and as demonstrated in research. Clinical EFT identifies 48 distinct techniques described in the manual and supplementary materials (www.ClinicalEFT.com).

\section{APA Standards, Fidelity, and Implementation}

The APA standards were developed in a series of papers (Chambless et al., 1996; Chambless et al., 1998, Chambless \& Hollon, 1998). Methods demonstrating efficacy according to certain criteria, such as two high-quality studies performed by independent investigators finding the method statistically superior to a placebo or another method, are said to be "efficacious." Methods that meet lesser standards are classified as "probably efficacious."

The APA standards may be summarized as comprising seven essential criteria (Energy Psychology Journal, 2012). Studies cannot be measured in order to determine if the method under investigation is "empirically validated" unless they meet all seven. Chambless \& Hollon (1998) also list additional criteria that may be divided into two further gradations: "highly desirable” and "desirable” (Energy Psychology Journal, 2012). The seven essential criteria are:

1) Randomized controlled trials (RCTs) - subjects were randomly assigned to the treatment of interest condition or to one or more comparison conditions.

2) Adequate sample size to detect statistically significant ( $p$ $<.05$ or better) differences between the treatment of interest and the comparison condition(s) were used.

3) The population for which the treatment was designed and tested must be clearly defined through the use of diagnosis by qualified clinicians, through cutoff scores on questionnaires that are reliable and valid, through interviews identifying the focus of the study's interest, or through some combination of these.

4) Assessment tools must have demonstrated reliability and validity in previous research.

5) Any interview assessments were made by interviewers who were blind to group assignment.

6) Treatment manuals that make clear the nature of the treatment being tested were used. If the treatment was relatively simple, it could be described in the procedure section of the journal article presenting the experiment, in lieu of a treatment manual.

7) The paper reporting the study provided enough data that the study's conclusions can be reviewed for appropriateness, including sample sizes, use of instruments that detect changes targeted by the study's design, and magnitude of statistical significance.

Studies of efficacious or probably efficacious therapies are required to demonstrate "statistically significant" results, meaning that there is less than 1 possibility in 20 that the results are due to chance (Criterion \#2). This meaning of the word "significance" as in "demonstrating statistically significant results" is expressed in research statistics as $p<.05$, or a probability of $20 \%$ (i.e., .05) that the results are due to chance. The term "highly significant" is often used to refer to studies with outcomes showing that there is less than 1 possibility in 1000 that the results are due to chance, or $p<.001$.

These APA criteria then are a stable, defined, published set of standards by which the efficacy of a therapeutic technique may be judged. When that technique is then translated into training, certification, and clinical practice, these criteria provide reasonable assurance that the method as practiced in the field is the method that has been validated in research.

\section{EFT as an Empirically Validated Treatment}

Having defined Clinical EFT and identified the set of standards upon which measurement of efficacy is based, we can now examine the evidence base that supports the efficacy of EFT. For this report, a literature search for English-language papers was performed using MEDLINE/PubMed, PsycINFO, Google Scholar, and references from the retrieved papers. Papers "in press" were obtained from professional organizations in the field. The search is current through May of 2013. Chronologically, the earliest group of EFT studies performed were outcome studies that asked the question, "Are participants better off after treatment?" Outcome studies of Clinical EFT can be grouped into three primary categories. These examine efficacy for:

- Psychological conditions such as PTSD, phobias, depression, and anxiety;

- Physiological problems such as pain and autoimmune conditions;

- Performance in sports, business, and academic pursuits.

This paper also summarizes the key research on the physiological mechanisms of action of Clinical EFT, showing how EFT works in the body to effect change. These studies, rather than measuring whether treatment benefits patients, ask the questions characteristic of basic science, such as "How does this treatment work?" and "What is going on in the body as a result of this treatment?” The final group of studies reviewed investigate EFT's application to performance issues such as public speaking anxiety as well as sports performance. We will also investigate whether EFT's somatic component, tapping with the fingertips on acupressure points, is an inert placebo or an active ingredient in the results obtained. Finally, we will derive the meaning of this whole body of work and extend it to show the next steps in EFT research, such as data gathering via smartphone apps, patient tolerance trials, large group studies, and EFTs application in primary care settings.

\section{Psychological Health Outcome Studies}

Clinical EFT has met APA standards as an "efficacious" or "probably efficacious" treatment for a number of conditions, including anxiety, depression, phobias, and PTSD (Feinstein, 
2012). Since RCTs are regarded as the Gold Standard of research, and are the type of experimental design usually used to evaluate a therapy against APA standards, only RCTs are listed in this section on mental health outcomes. There have been many other studies of EFT for these mental health conditions that were not RCTs, and some of these are referenced outside of this section on psychological health.

Anxiety. EFT has shown efficacy in several RCTs of anxiety. In one study, students with fear of public speaking received a 45-minute EFT session and improved significantly (Jones, Thornton, \& Andrews, 2011). In another, high school students with test anxiety were evaluated before their university entrance exams (Sezgin \& Özcan, 2009). Those who learned EFT improved significantly. A control group was taught progressive muscular relaxation. The improvement in the EFT group was significantly greater than that of the control group.

Other studies have also shown statistically significant reductions in anxiety in a variety of populations. A study of fibromyalgia sufferers found significant improvements in anxiety (Brattberg, 2008), as have studies of veterans and hospital patients with PTSD (Church, 2013b; Karatzias et al., 2011). An RCT of university students with test anxiety found significant improvements after both EFT and diaphragmatic breathing (DB) following EFT treatments (Jain \& Rubino, 2012). EFT was compared to cognitive behavioral therapy (CBT) in an RCT with female trauma survivors in the Congo (Nemiro, 2013). EFT was found to be as efficacious as CBT in reducing symptoms of anxiety, depression, and PTSD.

Three studies of anxiety fail to meet one or more APA standards. An RCT of psychological conditions in participants in a weight loss program found reductions in anxiety that closely approached significance $(p<.053)$ but did not meet the required $p<.05$ threshold (APA criterion \#2; Stapleton, Church, Sheldon, Porter, \& Carlopio, 2013). Benor, Ledger, Toussaint, Hett, and Zaccaro (2010) found significant reductions in anxiety in university students, but class scheduling conflicts among participants prevented true randomization (APA criterion \#1). Waite and Holder (2003) compared EFT to two sham tapping interventions and a non-tapping control group. However, the RCT failed to use valid and reliable assessments (APA Criterion \#4), failed to apply EFT with fidelity to the manual (APA criterion \#6), and failed to recognize that the "sham" points selected by the investigators were in fact actual acupressure points (APA criterion \#6). These errors resulted in all three tapping groups improving relative to the non-tapping group.

Depression. RCTs in which depression was measured before and after EFT have demonstrated large drops in depressive symptoms. A study examining college students with high levels of depression ("clinical" depression as measured by the Beck Depression Inventory) found that they were in the "normal" range after EFT (Church, De Asis, \& Brooks, 2012). The fibromyalgia study also found significant improvements in depression (Brattberg, 2008), as did the studies of hospital patients and veterans with PTSD (Karatzias et al., 2011; Church, 2013b). An RCT of weight loss program participants also found significant reductions in depressive symptoms (Stapleton, Church, Sheldon, Porter, \& Carlopio, 2013). The study of Congolese female trauma survivors also found EFT to be efficacious for depression when compared to CBT (Nemiro, 2013).

Phobias. Three RCTs have examined the effects of EFT on phobias and found that a single session is usually enough to resolve a phobia (Wells, Polglase, Andrews, \& Carrington,
2003; Baker \& Siegel, 2010; Salas, Brooks, \& Rowe, 2011). All three studies included a follow-up period and found that the phobic responses of participants remained significantly lower than before treatment.

PTSD. EFT has been studied as a treatment for clinical PTSD in three RCTs. One, in a population of 59 war veterans, found that PTSD symptoms dropped into the "normal" range after six sessions of EFT and remained that way on follow-up (Church et al., 2013). A hospital in Britain's National Health Service (NHS) compared EFT to another efficacious treatment, Eye Movement Desensitization and Reprocessing (EMDR), and found that both treatments normalized PTSD in an average of four sessions (Karatzias et al., 2011). An RCT of abused male teenagers found, on follow-up, that their PTSD symptoms had been resolved in a single EFT session (Church, Piña, Reategui, \& Brooks, 2011). When EFT was taught to groups of Congolese women with PTSD, EFT's efficacy was found to be comparable to that of CBT (Nemiro, 2013). As with most PTSD studies, Nemiro (2013) used the PTSD Checklist or PCL to evaluate symptoms (Ruggiero, Del Ben, Scotti, \& Rabalais, 2003). Studies with veterans usually use the military version of the same instrument, the PCL-M (Weathers, Huska, \& Keane, 1991).

Data from Church et al. (2013) were analyzed to determine if telephone sessions produced the same symptom reductions as office visits (Hartung \& Stein, 2012). While 67\% of veterans were subclinical after phone sessions, a significantly larger percentage of the sample recovered after office sessions. A further substudy based on Church et al. (2013) examined the performance of life coaches compared to licensed mental health professionals (Stein \& Brooks, 2011). It found larger reductions in symptoms in veterans treated by licensed practitioners, though the difference did not rise to the level of statistical significance. These analyses indicate the utility of EFT when delivered over the telephone, and by practitioners with very basic levels of training.

\section{Physiological Issues: Pain, Weight Loss, Cravings, and Physical Symptoms}

The studies of EFT for physical symptoms include a range of experimental designs, with both RCTs and studies without a control group ("uncontrolled" studies). The latter "within-subjects" studies use subjects as their own controls, comparing their symptom levels before and after EFT.

Pain and physical symptoms. Veterans were found to experience significant drops in physical pain after EFT (Church, 2013b), as were fibromyalgia sufferers (Brattberg, 2008). When PTSD symptoms were remediated in veterans, symptoms of traumatic brain injury (TBI) were reduced by $41 \%(p<.0021$; Church \& Palmer-Hoffman, 2013). An RCT of patients with tension headaches performed at the Red Cross Hospital in Athens found that the frequency and intensity of their headaches dropped by more than half after EFT, and other physical symptoms improved ( $p<.001$; Bougea et al., 2013). Uncontrolled studies and case reports showed improvement in a variety of conditions.

One study examined symptoms in 216 health care workers such as doctors, nurses, chiropractors, psychotherapists, and alternative medicine practitioners who attended a one-day EFT workshop at one of five professional conferences (Church \& Brooks, 2010). They experienced a $68 \%$ drop in physical pain 
$(p<.001)$. Though this was an uncontrolled study, it examined the five different groups separately, making it, in effect, five small studies. In addition, EFT was delivered by two different practitioners. Despite these disparities, all five groups showed similar results.

EFT was adopted by a clinic in Britain's NHS, which performed a "service evaluation" in order to determine the acceptance of EFT by patients and its success in reducing symptoms. This study found a significant improvement in anxiety, with a mean treatment time frame of eight sessions. It also found a significant improvement in overall psychological health and physical functioning (Stewart et al., 2013).

A pilot study of psoriasis symptoms also showed improvement in skin problems (Hodge \& Jurgens, 2011). Other authors report success with victims of motor accidents (Burk, 2010), dyslexia (McCallion, 2012), seizure disorders (Swingle, 2010), and TBI (Craig, Bach, Groesbeck, \& Benor, 2009).

Three studies have examined the effect on insomnia after EFT. The veterans PTSD study referenced above (Church et al., 2013) found a significant improvement in insomnia scores, with mean values dropping from the clinical range to the subclinical range $(p<.001)$. A pilot study of 10 geriatric patients with insomnia noted a similar reduction in insomnia, along with decreases in anxiety and depression, and an increase in life satisfaction (Lee, Suh, Chung, \& Kim, 2011). This led to an RCT conducted with 20 participants that compared EFT to an active control, Sleep Hygiene Education (Lee \& Kim, 2013). It demonstrated significant reductions in depression and insomnia. Insomnia is related to stress and to the regulation of the autonomic nervous system; the improvements found in these studies demonstrate the association between a reduction in stress symptoms and decreases in insomnia.

Weight loss and cravings. Studies have examined the use of EFT for weight loss and food cravings. An RCT found that EFT improved restraint (Stapleton, Sheldon, Porter, \& Whitty, 2011) and that, in the year following an EFT weight loss program, participants lost an average of 11.1 pounds (Stapleton, Sheldon, \& Porter, 2012). An uncontrolled study of clients in a 6 -week online weight loss program found a 12-pound weight reduction during the 6 weeks of the program, followed by a further 3-pound drop in the ensuing 6 months $(p<.001$; Church \& Wilde, 2013). In the health care workers study (Church \& Brooks, 2010) summarized previously, cravings for substances such as chocolate, sweets, and alcohol were reduced by $83 \%$ ( $p$ $<$.001). Group application of EFT was also found to reduce psychological symptoms such as anxiety in a group self-identified with addiction issues (Church \& Brooks, 2013b). A review found that EFT could also be useful as an adjunctive therapy for weight loss (Sojcher, Perlman, \& Fogerite, 2012).

\section{Sports and Professional Performance}

Mental health studies usually measure reductions in conditions such as anxiety, depression, and PTSD. They typically use reliable and valid assessments, such as the Beck Depression Inventory (BDI; Beck, Steer, \& Carbin, 1988), the Beck Anxiety Inventory (BAI; Fydrich, Dowdall, \& Chambless, 1992), the Fear Questionnaire (FQ; Mavissakalian, 1986), or the Hospital Anxiety and Depression Scale (HADS; Zigmond \& Snaith, 1983), as called for in APA standards, to measure symptom levels before and after treatment, to determine if they decrease. The focus of performance studies is different. They take indi- viduals who are already performing at a certain level, and seek to determine if their level of performance can be increased. Rather than a decrease in, for example, anxiety, they seek to measure an increase in, for example, confidence.

Two RCTs have examined EFT's efficacy for sports performance. One measured the difference in basketball free throw percentages between an EFT and a placebo control group and found a performance difference of $38 \%$ after a brief session (Church, 2009; Baker, 2010). Another found similar benefits for soccer free kicks (Llewellyn-Edwards \& Llewellyn-Edwards, 2012). A case study of golf performance found stressrelated errors decreasing after EFT (Rotherham, Maynard, Thomas, Bawden, \& Francis, 2012). A 20-minute EFT session was found to increase confidence and decrease anxiety in an uncontrolled study of female college-aged athletes (Church \& Downs, 2012).

Several studies summarized in the previous paragraphs examined the application of EFT to professional performance issues such as public speaking anxiety and test anxiety, and found improvements (Jones, Thornton, \& Andrews, 2011; Sezgin \& Özcan, 2009; Benor et al., 2009). Fox and Malinowski (2013) examined positive and negative emotions relating to academic study in a population of undergraduates, and found significant increases in enjoyment and hope, and decreases in anger and shame. The NHS service evaluation performed by Stewart et al. (2013) examined patient self-esteem using the Rosenberg Self-Esteem Scale (Rosenberg, 1989) and mental well-being using the Warwick-Edinburgh Mental Wellbeing Scale (University of Warwick and University of Edinburgh, 2012). It found that both mental well-being and self-esteem improved significantly $(p<.001)$. A study of university students preparing for exams found that EFT reduced their anxiety, and improved their test scores (Boath, Stewart, \& Carryer, 2013). Nursing students had reduced stress 4 weeks after learning EFT $(p<.005)$, and also exhibited decreases in both the state of anxiety, and the character trait of anxiety $(p<.05$; Patterson, 2013). Taken as a whole, this body of research indicates EFT's robust ability to reduce anxiety, whether it is occasioned by athletic, public speaking, or academic performance stress.

\section{Physiological Mechanisms of Action}

Outcome studies, which compare patient results before and after treatment, are clearly the most clinically important type of research. However, while showing that a treatment works allows it to be designated as an "evidence-based" practice, showing how and why it works allows us to understand the physiological changes that underlie its clinical benefits.

Three studies have used electroencephalogram (EEG) to examine the brain wave frequencies of participants before and after EFT. These studies provide us with objective physiological evidence, as opposed to the type of subjective self-report characteristic of mental health studies that use pen-and-paper assessments. Swingle, Pulos, and Swingle (2004) compared the EEG readings of auto accident victims before and after they learned EEG, and found a reduction in the frequencies associated with PTSD. Lambrou, Pratt, and Chevalier (2003) used acupressure tapping with claustrophobics, comparing them with a non-claustrophobic group, and found an increase in theta EEG frequencies associated with relaxation after treatment. Using electromyography (EMG), they also found significant relaxa- 
tion of the trapezius muscle. Claustrophobic subjects declined significantly in anxiety as well, with gains maintained on 2-week follow-up. Swingle (2010) found EFT to be beneficial in the treatment of seizure disorders. These three studies all reinforce the body of work in acupuncture that uses fMRI to demonstrate regulation of the fear centers of the brain (reviewed by Feinstein, 2010).

If EFT is regulating the body's stress response and the hypothalamus-pituitary-adrenal (HPA) axis, then it is also logical to look for changes in stress hormones such as norepinephrine (adrenaline) and cortisol. A triple-blind study examined the cortisol levels of 83 normal subjects before and after an hour of EFT (Church, Yount, \& Brooks, 2012). A control group received talk therapy while a second control group simply rested. Comparison of the three groups revealed significant reductions in cortisol in the EFT group compared to the other two groups $(p<.03)$. The overall severity of psychological symptoms dropped by $50.5 \%$ in the EFT group $(p<.001)$. This study demonstrated a significant relationship between the reduction in psychological conditions such as anxiety and depression, and cortisol. Improvements in mental health after therapy can be reflected in reduced levels of cortisol and regulation of the genes that code for such hormones (Feinstein \& Church, 2010). Scientists studying epigenetics emphasize the role stress and emotion plays in gene expression (Jirtle \& Skinner, 2007; Church, 2010b; Fraga et al., 2005; Eley \& Plomin, 1997).

\section{Is Acupoint Tapping an Active Ingredient in EFT?}

EFT's “Setup Statement” is an essential part of the "Basic Recipe.” The Setup Statement has two parts. One is a statement of the client's presenting problem, and clients are instructed to focus on the problem by saying something like, "Even though I have this problem..." while tapping on a specified acupressure point. They repeat the name of the problem while tapping on the other points. This focus on the problem is reminiscent of the exposure techniques practiced in Prolonged Exposure (PE) and other exposure therapies. The second half of the Setup Statement directs the client toward acceptance of conditions as they are: “... I deeply and completely accept myself.” This cognitive reframe is akin to the techniques used in cognitive therapies, which seek to modify dysfunctional client cognitions and emotional responses to events. In a review of therapies for PTSD, the US government's Institute of Medicine found that therapies that use exposure and cognitive shift were efficacious (Institute of Medicine, 2007). EFT's Setup Statement draws from elements of these two established therapies.

The third ingredient used by EFT is tapping on points used in acupuncture and acupressure (acupoints). Is this component of EFT an active ingredient, or is EFT's efficacy dependent solely on the exposure and cognitive components it shares with other therapies?

Fox and Malinowski (2013) sought to answer the question of whether tapping is an active ingredient or an inert placebo. Their study examined mindfulness, and study-related positive and negative emotions in an RCT of 20 undergraduates using the Achievement Emotions Questionnaire (Pekrun, Goetz, Frenzel, Barchfeld, \& Perry, 2011). The EFT group received the Basic Recipe as described in The EFT Manual. The control group received the cognitive and exposure elements of the Basic Recipe but without acupoint tapping. Instead, they received an active control of diaphragmatic breathing (DB) in its place. The intervention lasted 40 minutes, and participants were reassessed 7 days later. Significant improvement in study-related positive emotions such as enjoyment and hope was found, along with decreases in negative emotions such as anger and shame. No change in mindfulness was detected.

This indicates that EFT's acupoint stimulation is an active ingredient. This finding supports studies that use fMRI to measure the effects of acupuncture on the areas of the brain associated with fear (Hui et al., 2005; Fang et al., 2009; Napadow et al., 2007). These studies uniformly report acupuncture to produce rapid regulation of these brain regions. They are also consistent with the studies that use EEG (electroencephalogram) to evaluate EFT. They find that EFT reduces the brain wave frequencies associated with stress or amplifies those associated with relaxation, as well as producing other beneficial physiological changes (Swingle, Pulos, \& Swingle, 2004; Lambrou, Pratt, \& Chevalier, 2003; Swingle, 2010). When the established protocols drawn from exposure and cognitive therapies are paired with acupressure, their effects appear to be enhanced. It is probable that the amygdala and other fear-processing centers of the nervous system are being regulated, as stress-laden emotions are calmed (Phelps \& LeDoux, 2005).

\section{EFT as Group Therapy}

During the early development of EFT, practitioners reported lower levels of stress and burnout than they had experienced previously, while administering therapies other than EFT to clients. This led to the hypothesis that tapping on oneself while demonstrating tapping to others, or witnessing tapping on others while tapping on oneself, diminished distress. This phenomenon is known as "Borrowing Benefits" (Craig, 2010). A series of studies has measured the efficacy of Borrowing Benefits for psychological and physical symptoms.

The first such study was performed by Rowe (2005). Rowe examined the psychological symptom levels of participants in a weekend EFT workshop using a valid and reliable assessment, the Symptom Assessment 45 (SA-45). The SA-45 has two general scales for the breadth and depth of psychological distress, as well as measuring levels of nine common conditions such as anxiety and depression. Rowe found a reduction in both general and specific scales, with participant gains maintained on follow-up.

The health care workers study cited previously (Church \& Brooks, 2010) also utilized the SA-45, with similar results. Most participant gains were maintained at 3-month follow-up $(p<.0001)$. The physical pain of subjects was reduced by $68 \%$, and their cravings were reduced by $83 \%$ (both $p<.0001$ ). At follow-up, this study also compared the relative symptom levels of participants who had used EFT frequently with those who had not. It found greater improvements in more frequent users. Another study also found that EFT was effective in groups taught by a variety of trained practitioners (Palmer-Hoffman \& Brooks, 2011), suggesting that the improvements were due to the EFT method itself, rather than the unique gifts of any one practitioner. The addiction study summarized previously found similar improvements from Borrowing Benefits, with durable gains (Church \& Brooks, 2013b).

PTSD symptoms were examined in a study of 218 veterans and spouses who attended 7-day group retreats (Church \& Brooks, 2013a). On pretest, $82 \%$ of veterans and $29 \%$ of 
spouses met the criteria for clinical levels of PTSD symptoms. After the retreat, at 6-week follow-up, only $28 \%$ of veterans and $4 \%$ of spouses were PTSD-positive $(p<.001)$. The study compared the results of five such retreats, reporting in effect the results of five individual substudies. Similar symptom declines were noted in all five groups. This study points to EFT's ability to reduce PTSD symptoms in large groups of people simultaneously.

Though these were uncontrolled studies, several RCTs also utilized a group therapy design. The study of college students with depression (Church, De Asis, \& Brooks, 2012) offered the EFT intervention in four group counseling sessions. The study of depression in weight loss subjects also taught participants EFT in group classes (Stapleton, Church, Sheldon, Porter, \& Carlopio, 2013). Two of the studies of depression (Jones, Thornton, \& Andrews, 2011; Sezgin \& Özcan, 2009) also provided EFT instruction to participants as a group. In two of the studies of sports performance, Church (2009) and LlewellynEdwards \& Llewellyn-Edwards (2012), the EFT cohort received at least part of the intervention as a group. EFT was also provided in groups of 10 in the Congo RCT of traumatized females, and found to be as effective as CBT in reducing PTSD, anxiety, and depression (Nemiro, 2013). The insomnia RCT also administered both EFT and the active control in group format (Lee \& Kim, 2013).

These studies are notable in that significant reductions in symptoms occurred when EFT was delivered as group therapy, as opposed to individual counseling. If EFT is able to consistently reduce psychological symptoms by $45 \%$, as the five groups treated in Church and Brooks (2010) demonstrate, EFT may be unusually effective when delivered to groups. The number of recent Middle East war veterans with PTSD is estimated at a minimum of 500,000; according to a September 2012 report from the Department of Veterans Affairs, almost $30 \%$ of the 834,463 Iraq and Afghanistan War veterans treated at VA hospitals and clinics over the course of the previous decade have been diagnosed with PTSD (Veterans Health Administration, 2012). This is in addition to the estimated 479,000 Vietnam veterans with PTSD (Dohrenwend et al., 2006). Each veteran with PTSD is estimated to cost society $\$ 1,400,000$ (Kanter, 2007), implying a social cost of about a trillion dollars to treat these two cohorts. Therapies such as Clinical EFT, which produce symptom reductions without the need for lengthy individual courses of psychotherapy or chronic use of prescription drugs, are simultaneously efficient and cost effective.

\section{Simultaneous Symptom Reduction}

Most psychological research seeks to isolate a single condition and excludes multiple diagnoses (Seligman, 1995). For instance, a study of PTSD might exclude clients with comorbid major depression or generalized anxiety. EFT's client-centered approach focuses on the distress as experienced by the client, rather than the primacy of diagnosis by the therapist. EFT is often successful at treating several diagnoses simultaneously. An RCT found that EFT was efficacious for PTSD in six sessions, with $86 \%$ of veterans subclinical after six sessions (Church et al., 2013). Data from the same study were later analyzed to reveal that EFT simultaneously reduced anxiety and depression (Church, 2013b). Furthermore, EFT's pervasive treatment effects encompass both psychological and physio- logical symptoms. Analysis showed that TBI symptoms diminished significantly and continued to decline throughout the follow-up period, cumulatively reducing 41\% from pretest baseline ( $p<.0021$; Church \& Palmer-Hoffman, 2013). Physical pain was reduced by $41 \%$ ( $p<.0001$; Church, 2013b). Two early pilot studies of EFT for PTSD found that not only did PTSD symptoms drop significantly, but anxiety and depression symptoms also declined in parallel (Church, 2010a; Church, Geronilla, \& Dinter, 2009). EFT is thus extraordinarily efficient, addressing multiple symptom domains simultaneously. Scholars have noted that most clients present with a complex of disorders, rather than a single one (Gorman, 1998).

\section{Safety}

EFT also appears to be safe when administered by a therapist or life coach, or self-administered. Therapists treating victims of childhood sexual abuse preferred energy psychology treatments such as EFT over talk therapy because they found the risk of abreaction low with the former (Schulz, 2009). Mollon (2007) reports a general reduction of client distress during acupoint tapping, while Flint, Lammers, and Mitnick (2005) remark on the absence of abreactions during energy psychology treatments. Most studies of EFT have been performed after Institutional Review Board (IRB) review. IRB procedures require that studies be designed and conducted in a manner that protects human subjects, including a requirement that participants be monitored for adverse events. Cumulatively, over 1000 subjects have participated in trials of EFT without a single adverse event being reported, indicating a high degree of safety.

\section{Research Reviews}

Many review articles about EFT have been written. Reviews systematically gather the evidence for a method and ask, "What does this mean?" and "What does this body of research, taken as a whole, suggest?" Notable reviews include those of Feinstein (2012) on the evidence for the efficacy of acupoint tapping, Lane (2009) on the physiological mechanism of action of energy therapies, Feinstein (2010) for energy psychology as applied to PTSD, Feinstein and Church (2010) showing how successful psychotherapy can be measured physiologically, Church and Feinstein (2012) emphasizing that EFT in clinical practice is fast and effective, and Feinstein (2008) for the effects of acupoint tapping for survivors in disaster zones. The body of primary research summarized and evaluated in these review articles, as well as the studies reviewed previously, allow us to draw several conclusions about Clinical EFT:

1) It reduces symptoms for a variety of psychological conditions including phobias, PTSD, anxiety, and depression.

2) It improves physical symptoms such as pain, and autoimmune conditions such as psoriasis and fibromyalgia.

3) It aids in reducing cravings and promoting weight loss.

4) It produces physiological regulation of the autonomic nervous system and the HPA axis.

5) It can simultaneously reduce a range of psychological conditions, e.g., diminishing anxiety and depression along with PTSD.

6) It can simultaneously reduce both psychological and physiological problems, e.g., fibromyalgia or TBI concurrent with PTSD, anxiety, and depression.

7) It is safe, both when self-administered and when adminis- 
tered by others.

8) It is efficient and cost effective, showing efficacy when delivered to both groups and individuals.

9) It works quickly. Treatment time frames range from one session for phobias to six sessions for PTSD.

10) Early evidence points to its efficacy when it is delivered online.

11) It can play a useful part in early intervention following human-caused and natural disasters.

\section{Future Research Directions}

Having met APA criteria as an "efficacious" or "probably efficacious" treatment for several conditions, such as phobias, PTSD, anxiety, and depression, what are the next steps for EFT research?

Larger trials. Most of the RCTs conducted have had a small number of participants. Because of EFT's robust treatment effects, studies are able to achieve statistical significance with a small number of participants. However, confirmation with trials involving 100 or more participants per group will provide strata of information not possible with smaller groups, such as whether EFT is more effective with certain demographics.

Institutional trials. Most studies have been conducted in outpatient settings by private foundations. Studies within institutions such as large hospitals will provide a framework for institution-wide implementation of EFT.

Online application. There are only two studies to date in which EFT has been delivered online (Brattberg, 2008; Church \& Wilde, 2013). The results of these studies were encouraging, showing improvements in depression and anxiety in both fibromyalgia sufferers and weight loss program participants. Yet this early research only hints at the possibilities. Much more work is needed to determine how EFT can be applied effectively in online programs. Emerging technologies like smartphones allow EFT to be used portably during times of heightened stress. There are several EFT iPhone apps, but none has been subject to experimental testing. Given the low cost of delivery and ease of automated data gathering, online trials of EFT are a logical next step.

Medical applications. Only one of the trials of EFT for physiological functioning, the cortisol study, had a large number of participants (Church, Yount, \& Brooks, 2012). The three EEG studies (Swingle, Pulos, \& Swingle, 2004; Lambrou, Pratt, \& Chevalier, 2003; Swingle, 2010) all had a small number of participants, yet all four studies taken together point to EFT's potential as a medical intervention. Cortisol is known to correlate with HPA arousal, heart rate variability (HRV), and other stress-regulation systems in the body. Depression and anxiety are associated with many diseases. Yet the existing studies of EFT only hint at EFT's potential to affect the course of such diseases. Medical trials could explicitly identify EFT's utility as a medical intervention for conditions such as hypertension, diabetes, cancer, and cardiac events, all of which are stressrelated. Such studies could ask research questions like: Does EFT:

Speed wound healing?

Reduce cardiac events markers like C-reactive protein?

Increase circulatory cytokines?

Reduce hypertension?

Reduce chronic pain?

Slow aging by decreasing telomere loss?
Downregulate oncogenes?

Raise levels of cell repair hormones like dehydroepiandrosterone (DHEA)?

Promote healthy balances of neurotransmitters such as serotonin and dopamine?

If EFT is able to demonstrate any of these effects, it can be introduced into primary care as an auxiliary behavioral treatment that is safe and free of side effects.

Patient tolerance studies. Behavioral interventions such as meditation, yoga, diaphragmatic breathing, and EFT are rarely integrated into regular patient care. Research can determine how to effectively introduce patients to EFT and encourage compliance with a health-promoting stress-reduction regimen. Might outpatients benefit from using an EFT app loaded into their smartphones before an appointment? Might patients preparing for surgery benefit by being taught EFT as a stressmanagement tool to use before and after a procedure? These and other questions could be answered by research aimed at improving patient care.

Group scale studies. EFT is notable in its ability to improve symptoms when delivered to groups. However, the optimal group size has not yet been tested. The group evaluated by Rowe (2005) comprised 259 participants, with 102 providing complete data, while some of the groups in other studies have been as small as 10 (Church \& Brooks, 2010). What is the minimum size to produce a group effect? What is the optimum size for each condition? Is there a group size at which the effects diminish? Research that answers these questions of scale will assist institutions using group therapy to optimize their use of Clinical EFT.

Pervasive symptom focus. While research has tended to isolate conditions such as chronic pain or depression, EFT's ability to reduce both psychological and physiological symptoms in tandem might push research toward measuring client-centered reports of symptom clusters, and away from reliance solely on observer-rated clinical diagnoses.

Biomarkers for psychological change. Feinstein and Church (2010) advocate salivary cortisol testing as an objective measure for the efficacy of psychotherapy. A holistic approach means that physiological markers might become a standard measure of efficacy. As cheaper and simpler gene assays become available, the effect of EFT and other therapies on gene expression might be measured by these objective biomarkers.

Healthcare cost reductions. If symptoms of psychological conditions such as anxiety and depression reduce after EFT treatment, what is the impact on healthcare costs? Presumably they decline, but by how much? The symptom reductions noted in the research literature suggest that widespread implementation of EFT will have a substantial impact on healthcare costs, but this has not yet been quantified. Studies examining the costs before and after EFT implementation in settings such as hospitals and organizations will yield quantifiable measures of the effects of treatment.

\section{The Maturing Field of Clinical EFT}

Clinical EFT, as validated in many RCTs and outcome studies, has established itself as an efficacious treatment for both psychological and physical conditions. Clinical EFT enjoys a large and growing body of research that has validated it as an "evidence-based" practice that is safe, fast, reliable, and costeffective. Clinical EFT is supported by professional training 
programs that teach practitioners to deliver the method as validated by research based on The EFT Manual (Craig, 2010; Church, 2013a). Clinical EFT is elucidated in depth in The Clinical EFT Handbook: A Definitive Resource for Practitioners, Scholars, Clinicians and Researchers (Church \& Marohn, 2013). The literature demonstrates sufficient clinical benefit from EFT to argue for its adoption as a front-line primary care intervention within a wide variety of settings and populations.

\section{REFERENCES}

Baker, A. H. (2010). A re-examination of Church's (2009) study into the effects of Emotional Freedom Techniques (EFT) on basketball free-throw performance. Energy Psychology: Theory, Research, \& Treatment, 2, 39-44.

Baker, A. H., \& Siegel, M. A. (2010). Emotional freedom techniques (EFT) reduces intense fears: A partial replication and extension of Wells et al. Energy Psychology: Theory, Research, and Treatment, 2, 13-30.

Beautler, L. E., Norcross, J. C., \& Beutler, L. E. (2005). Evidencebased practices in mental health: Debate and dialogue on the fundamental questions. Washington DC: American Psychological Association.

Beck, A. T., Steer, R. A., \& Carbin, M. G. (1988). Psychometric properties of the beck depression inventory: Twenty-five years of evaluation. Clinical Psychology Review, 8, 77-100. doi:10.1016/0272-7358(88)90050-5

Benor, D. J., Ledger, K., Toussaint, L., Hett, G., \& Zaccaro, D. (2009). Pilot study of emotional freedom techniques, wholistic hybrid derived from eye movement desensitization and reprocessing and emotional freedom techniques, and cognitive behavioral therapy for treatment of test anxiety in university students. Explore: The Journal of Science and Healing, 5, 338-340. doi:10.1016/j.explore.2009.08.001

Boath, E., Stewart, A, \& Carryer, A. (2013). Tapping for success: A pilot study to explore if emotional freedom techniques (EFT) can reduce anxiety and enhance academic performance in university students. Innovative Practice in Higher Education, 1.

Bougea, A. M., Spandideas, N., Alexopoulos, E. C., Thomaides, T., Chrousos, G. P., \& Darviri, C. (2013). Effect of the emotional freedom technique on perceived stress, quality of life, and cortisol Salivary levels in tension-type headache sufferers: A randomized controlled trial. Explore: The Journal of Science and Healing, 9, 91-99. doi:10.1016/j.explore.2012.12.005

Brattberg, G. (2008). Self-administered EFT (emotional freedom techniques) in individuals with fibromyalgia: A randomized trial. Integrative Medicine: A Clinician's Journal, 7, 30-35.

Burk, L. (2010). Single session EFT (emotional freedom techniques) for stress-related symptoms after motor vehicle accidents. Energy Psychology: Theory, Research, and Treatment, 2, 65-71.

Callahan, R. J. (2001). The impact of thought field therapy on heart rate variability. Journal of Clinical Psychology, 57, 1153-1170. doi:10.1002/jclp.1082

Chambless, D. L., Sanderson, W. C., Shoham, V., Bennett Johnson, S., Pope, K. S., Crits-Christoph, P., \& McCurry, C. (1996). An update on empirically validated therapies. Clinical Psychologist, 49, 5-18.

Chambless, D., \& Hollon, S. D. (1998). Defining empirically supported therapies. Journal of Consulting and Clinical Psychology, 66, 7-18. doi:10.1037/0022-006X.66.1.7

Chambless, D., Baker, M. J., Baucom, D. H., Beutler, L. E., Calhoun, K. S., Crits-Christoph, P., \& Woody, S. R. (1998). Update on empirically validated therapies, II. Clinical Psychologist, 51, 3-16.

Church, D. (2010a). The treatment of combat trauma in veterans using EFT (Emotional Freedom Techniques): A pilot protocol. Traumatology, 16, 55-65. doi:10.1177/1534765609347549

Church, D. (2010b). Your DNA is not your destiny: Behavioral epigenetics and the role of emotions in health. Anti-Aging Therapeutics, 13, 35-42.

Church, D. (2013). The EFT mini-manual. Santa Rosa, CA: Energy
Psychology Press.

Church, D. (2013a). The EFT manual (3rd ed.). Santa Rosa, CA: Energy Psychology Press.

Church, D. (2013b). Pain, depression, and anxiety after PTSD symptom remediation in veterans. Explore: The Journal of Science and Healing, in Press.

Church, D., \& Brooks, A. J. (2010). The effect of a brief EFT (emotional freedom techniques) self-intervention on anxiety, depression, pain and cravings in healthcare workers. Integrative Medicine: A Clinician's Journal, 9, 40-44.

Church, D., \& Brooks, A. J. (2013a). CAM and energy psychology techniques remediate PTSD symptoms in veterans and spouses. Explore: The Journal of Science and Healing, in Press.

Church, D., \& Brooks, A. J. (2013b). The effect of EFT (emotional freedom techniques) on psychological symptoms in addiction treatment: A pilot study. International Journal of Scientific Research and Reports, 2.

Church, D., \& Downs, D. (2012). Sports confidence and critical incident intensity after a brief application of emotional freedom techniques: A pilot study. Sport Journal, 15.

Church, D., \& Feinstein, D. (2013). Energy psychology in the treatment of PTSD: Psychobiology and clinical principles. In T. Van Leeuwen, \& M. Brouwer (Eds.), Psychology of trauma (pp. 211-224). Hauppage, NY: Nova Science Publishers.

Church, D., \& Marohn, S. (2013). The clinical EFT handbook: A definitive resource for practitioners, scholars, clinicians and researchers. Santa Rosa, CA: Energy Psychology Press.

Church, D., \& Palmer-Hoffman, J. (2012). TBI symptoms improve after PTSD remediation with emotional freedom techniques. Presented at the Conference Veterans, Treatment, and Trauma, Omega Institute, Rhinebeck, New York, Submitted for Publication.

Church, D., \& Wilde, N. (2013). Emotional eating and weight loss following Skinny Genes, a six week online program. Reported at the annual conference of the Association for Comprehensive Energy Psychology (ACEP), Reston, VA.

Church, D., De Asis, M. A., \& Brooks, A. J. (2012). Brief group intervention using EFT (emotional freedom techniques) for depression in college students: A randomized controlled trial. Depression Research and Treatment, 2012, 1-7. doi:10.1155/2012/257172

Church, D., Geronilla, L., \& Dinter, I. (2009). Psychological symptom change in veterans after six sessions of EFT (Emotional Freedom Techniques): An observational study. International Journal of Healing and Caring, 9.

Church, D., Hawk, C., Brooks, A., Toukolehto, O., Wren, M., Dinter, I., \& Stein, P. (2013). Psychological trauma symptom improvement in veterans using EFT (emotional freedom techniques): A randomized controlled trial. Journal of Nervous and Mental Disease, 201, 153160. doi:10.1097/NMD.0b013e31827f6351

Church, D., Piña, O., Reategui, C., \& Brooks, A. (2012). Single session reduction of the intensity of traumatic memories in abused adolescents after EFT: A randomized controlled pilot study. Traumatology, 18, 73-79. doi:10.1177/1534765611426788

Church, D., Yount, G., \& Brooks, A. J. (2012). The effect of emotional freedom techniques (EFT) on stress biochemistry: A randomized controlled trial. Journal of Nervous and Mental Disease, 200, 891896. doi:10.1097/NMD.0b013e31826b9fc1

Craig, G. (2010). The EFT manual. Santa Rosa, CA: Energy Psychology Press.

Craig, G., \& Fowlie, A. (1995). Emotional freedom techniques: The manual. Sea Ranch, CA: Gary Craig.

Craig, G., Bach, D., Groesbeck, G., \& Benor, D. J. (2009). Emotional freedom techniques (EFT) for traumatic brain injury. International Journal of Healing and Caring, 9.

Dohrenwend, B. P., Turner, J. B., Turse, N. A., Adams, B. G., Koenen, K. C., \& Marshall, R. (2006). The psychological risks of Vietnam for U.S. veterans: A revisit with new data and methods. Science, 313, 979-982. doi:10.1126/science.1128944

Eley, T. C., \& Plomin, R. (1997). Genetic analyses of emotionality. Current Opinion in Neurobiology, 7, 279-284. doi:10.1016/S0959-4388(97)80017-7

Energy Psychology Journal (2012). Research-supported psychological 
treatments. http://energypsychologyjournal.org/div12

Fang, J., Jin, Z., Wang, Y., Li, K., Kong, J., Nixon, E. E., \& Hui, K. K.-S. (2009). The salient characteristics of the central effects of acupuncture needling: Limbic-paralimbic-neocortical network modulation. Human Brain Mapping, 30, 1196-1206. doi:10.1002/hbm.20583

Feinstein, D. (2008) Energy psychology in disaster relief. Traumatology, 14, 124-137. doi:10.1177/1534765608315636

Feinstein, D. (2009). Controversies in energy psychology. Energy Psychology: Theory, Research, Practice, Training, 1, 45-56.

Feinstein, D. (2010). Rapid treatment of PTSD: Why psychological exposure with acupoint tapping may be effective. Psychotherapy: Theory, Research, Practice, Training, 47, 385-402. doi:10.1037/a0021171

Feinstein, D. (2012). Acupoint stimulation in treating psychological disorders: Evidence of efficacy. Review of General Psychology, 16, 364-380. doi:10.1037/a0028602

Feinstein, D., \& Church, D. (2010) Modulating gene expression through psychotherapy: The contribution of non-invasive somatic interventions. Review of General Psychology, 14, 283-295. doi:10.1037/a0021252

Flint, G. A., Lammers, W., \& Mitnick, D. G. (2005). Emotional Freedom techniques: A safe treatment intervention for many trauma based issues. In J. Garrick, \& M. B. Williams (Eds.), Trauma treatment techniques: Innovative trends (pp. 125-150). New York: Routledge.

Food and Drug Administration (1998). Guidance for industry: Providing clinical evidence of effectiveness for human drug and biological products. Rockville, MD: US Department of Health and Human Services.

Fox, L., \& Malinowski, P. (2013). Improvement in study-related emotions in undergraduates following emotional freedom techniques (EFT): A single-blind controlled study. Energy Psychology: Theory, Research, and Treatment, in Press.

Fraga, M. F., Ballestar, E., Paz, M. F., Ropero, S., Setien, F., Ballestar, M. L., \& Esteller, M. (2005). Epigenetic differences arise during the lifetime of monozygotic twins. Proceedings of the National Academy of Sciences USA, 102, 10604-10609. doi:10.1073/pnas.0500398102

Fydrich, T., Dowdall, D., \& Chambless, D. L. (1992). Reliability and validity of the Beck Anxiety Inventory. Journal of Anxiety Disorders, 6, 55-61. doi:10.1016/0887-6185(92)90026-4

Google Trends (2013).

http://www.google.com/trends/explore\#q=eft\&cmpt=q

Gorman, J. M. (1998). Comorbid depression and anxiety spectrum disorders. Depression and Anxiety, 4, 160-168. doi:10.1002/(SICI)1520-6394(1996)4:4<160::AID-DA2>3.0.CO;2-J

Hartung, J., \& Stein, P. (2012). Telephone delivery of EFT (emotional freedom techniques) remediates PTSD symptoms in veterans: A randomized controlled trial. Energy Psychology: Theory, Research, \& Treatment, 4, 33-42.

Hodge, P. M., \& Jurgens, C. Y. (2011). A pilot study of the effects of Emotional Freedom Techniques in psoriasis. Energy Psychology: Theory, Research, \& Treatment, 3, 13-24.

Hui, K. K. S., Liu, J., Marina, O., Napadow, V., Haselgrove, C., Kwong, K. K., \& Makris, N. (2005). The integrated response of the human cerebro-cerebellar and limbic systems to acupuncture stimulation at ST 36 as evidenced by fMRI. NeuroImage, 27, 479-496. doi:10.1016/i.neuroimage.2005.04.037

Institute of Medicine, Committee on Treatment of Posttraumatic Stress Disorder (2007). Treatment of posttraumatic stress disorder: An assessment of the evidence. Washington DC: Institute of Medicine. http://www.nap.edu/catalog/11955.html

Jain, S., \& Rubino, A. (2012). The effectiveness of emotional freedom techniques (EFT) for optimal test performance: A randomized controlled trial. Energy Psychology: Theory, Research, and Treatment, 4, 13-24.

Jirtle, R. L., \& Skinner, M. K. (2007). Environmental epigenomics and disease susceptibility. Nature Reviews. Genetics, 8, 253-262. doi:10.1038/nrg2045

Jones, S., Thornton, J., \& Andrews, H. (2011). Efficacy of EFT in reducing public speaking anxiety: A randomized controlled trial.
Energy Psychology: Theory, Research, and Treatment, 3, 19-32.

Kanter, E. (2007). Shock and awe hits home. Washington DC: Physicians for Social Responsibility.

Karatzias, T., Power, K., Brown, K., McGoldrick, T., Begum, M., Young, J., \& Adams, S. (2011). A controlled comparison of the effectiveness and efficiency of two psychological therapies for posttraumatic stress disorder: Eye movement desensitization and reprocessing vs. emotional freedom techniques. Journal of Nervous and Mental Disease, 199, 372-378. doi:10.1097/NMD.0b013e31821cd262

Lambrou, P. T., Pratt, G. J., \& Chevalier, G. (2003). Physiological and psychological effects of a mind/body therapy on claustrophobia. Subtle Energies and Energy Medicine, 14, 239-251.

Lane, J. (2009). The neurochemistry of counterconditioning: Acupressure desensitization in psychotherapy. Energy Psychology: Theory, Research, and Treatment, 1, 31-44.

LeDoux, J. (2002). Synaptic self: How our brains become who we are. New York: Penguin.

Lee, J.-H, \& Kim, J. W. (2013). Randomized controlled trial for the evaluation of the effects of EFT-Insomnia (EFT-I) for the elderly. Master's Thesis, Korea: Kyung Hee University.

Lee, J.-H, Suh, H.-U., Chung, S.-Y, \& Kim, J. W. (2011). A preliminary study for the evaluation of the effects of EFT-I (EFT program for insomnia) for insomnia in the elderly. Journal of Oriental Neuropsychiatry, 22, 101-109.

Llewellyn-Edwards, T., \& Llewellyn-Edwards, M. (2012). The effect of emotional freedom techniques (EFT) on soccer performance. Fidelity: Journal for the National Council of Psychotherapy, 47, 14-21.

Mavissakalian, M. (1986). The fear questionnaire: A validity study. Behaviour Research and Therapy, 24, 83-85. doi:10.1016/0005-7967(86)90154-3

McCallion, F. (2012). Emotional freedom techniques for dyslexia. Energy Psychology: Theory, Research, and Treatment, 4, 35-46.

Melnyk, B. M., \& Fineout-Overholt, E. (2005). Making the case for evidence-based practice. Philadelphia, PA: Lippincott Williams \& Wilkins.

Mollon, P. (2007). Thought field therapy and its derivatives: Rapid relief of mental health problems through tapping on the body. Primary Care and Community Psychiatry, 12, 123-127.

Napadow, V., Kettner, N., Liu, J., Li, M., Kwong, K. K., Vangel, M., \& Hui, K. K. (2007) Hypothalamus and amygdala response to acupuncture stimuli in carpal tunnel syndrome. Pain, 130, 254-266. doi:10.1016/j.pain.2006.12.003

National Institute for Clinical Excellence (2009). How NICE clinical guidelines are developed: An overview for stakeholders, the public and the NHS.

http://www.nice.org.uk/media/62F/36/How_NICE_clinical_guideline s_are_developed_4th_edn_FIANL_LR.pdf

Nemiro, A. (2013). EFT vs CBT in the treatment of sexual gender based violence in the Democratic Republic of the Congo. Presented at the conference of the Association for Comprehensive Energy Psychology (ACEP), San Diego, CA.

Ornish, D., Magbanua, M. J. M., Weidner, G., Weinberg, V., Kemp, C., Green, C., \& Carroll, P. R. (2008). Changes in prostate gene expression in men undergoing an intensive nutrition and lifestyle intervention. Proceedings of the National Academy of Sciences USA, 105, 8369-8374. doi:10.1073/pnas.0803080105

Palmer-Hoffman, J., \& Brooks, A. J. (2011). Psychological symptom change after group application of emotional freedom techniques (EFT). Energy Psychology: Theory, Research, \& Treatment, 3, 3338.

Patterson, S. L. (2013). The effect of emotional freedom technique on stress and anxiety in nursing students. Presented at the conference of the Association for Comprehensive Energy Psychology (ACEP), San Diego.

Pekrun, R., Goetz, T., Frenzel, A. C., Barchfeld, P., \& Perry, R. P. (2011). Measuring emotions in students' learning and performance: The Achievement Emotions Questionnaire (AEQ). Contemporary Educational Psychology, 36, 36-48.

doi:10.1016/j.cedpsych.2010.10.002

Phelps, E. A., \& LeDoux, J. E. (2005). Contributions of the amygdala 


\section{D. $\mathrm{CHURCH}$}

to emotion processing: From animal models to human behavior. Neuron, 48, 175-187. doi:10.1016/j.neuron.2005.09.025

Rosenberg, M. (1989). Society and the adolescent self-image (Rev. ed.). Middletown, CT: Wesleyan University Press.

Rotherham, M., Maynard, I., Thomas, O., Bawden, M., \& Francis, L. (2012). Preliminary evidence for the treatment of type I "yips": The efficacy of the emotional freedom techniques. Sports Psychologist, 26, 551-570.

Rowe, J. E. (2005). The effects of EFT on long-term psychological symptoms. Counseling and Clinical Psychology, 2, 104-111.

Ruggiero, K. J., Del Ben, K., Scotti, J. R., \& Rabalais, A. E. (2003) Psychometric properties of the PTSD checklist-civilian version. Journal of Traumatic Stress, 16, 495-502. doi:10.1023/A:1025714729117

Salas, M. M., Brooks, A. J., \& Rowe, J. E. (2011). The immediate effect of a brief energy psychology intervention (Emotional Freedom Techniques) on specific phobias: A pilot study. Explore: The Journal of Science and Healing, 7, 255-260.

Schulz, P. (2009). Integrating energy psychology into treatment for adult survivors of childhood sexual abuse. Energy Psychology: Theory, Research, and Treatment, 1, 15-22.

Seligman, M. E. P. (1995). The effectiveness of psychotherapy: The consumer reports study. American Psychologist, 50, 965-974. doi:10.1037/0003-066X.50.12.965

Sezgin, N., \& Özcan, B. (2009). The effect of progressive muscular relaxation and Emotional Freedom Techniques on test anxiety in high school students: A randomized controlled trial. Energy Psychology: Theory, Research, and Treatment, 1, 23-30.

Sherman, J. J. (1998). Effects of psychotherapeutic treatments for PTSD: A meta-analysis of controlled clinical trials. Journal of Traumatic Stress, 11, 413-435. doi:10.1023/A:1024444410595

Sojcher, R., Perlman, A., \& Fogerite, S. (2012). Evidence and potential mechanisms for mindfulness practices and energy psychology for obesity and binge-eating disorder. Explore: The Journal of Science and Healing, 8, 271-276. doi:10.1016/j.explore.2012.06.003

Stapleton, P. B., Sheldon, T., \& Porter, B. (2012). Clinical benefits of Emotional Freedom Techniques on food cravings at 12-months follow-up: A randomized controlled trial. Energy Psychology: Theory, Research, and Treatment, 4, 1-12.

Stapleton, P., Church, D., Sheldon, T., Porter, B., \& Carlopio, C. (2013). Depression symptoms improve after successful weight loss with EFT (Emotional Freedom Techniques): A randomized controlled trial. Depression Research and Treatment, in Press.
Stapleton, P., Sheldon, T., Porter, B., \& Whitty, J. (2011). A randomised clinical trial of a meridian-based intervention for food cravings with six-month follow-up. Behaviour Change, 28, 1. doi:10.1375/bech.28.1.1

Stein, P., \& Brooks, A. J. (2011). Efficacy of EFT (emotional freedom techniques) provided by coaches vs. licensed therapists in veterans with PTSD. Energy Psychology: Theory, Research, \& Treatment, 3 , 11-17.

Stewart, A., Boath, E., Carryer, A., Walton, I., Hill, L., Phillips, D., \& Dawson, K. (2013). Can matrix reimprinting using EFT be effective in the treatment of emotional conditions? Energy Psychology: The ory, Research, \& Treatment, in Press.

Swingle, P. (2010). EFT in the neurotherapeutic treatment of seizure disorders. Energy Psychology: Theory, Research, \& Treatment, 2, 27-38.

Swingle, P. G., Pulos, L., \& Swingle, M. K. (2004). Neurophysiological indicators of EFT treatment of posttraumatic stress. Subtle Energies and Energy Medicine, 15, 75-86.

Traffic Estimate (2013). http://www.trafficestimate.com/eft.mercola.com

University of Warwick and University of Edinburgh (2012). The Warwick-Edinburgh Mental Well-being Scale (WEMWBS). http://www.healthscotland.com/documents/1467.aspx

Veterans Health Administration (2012). Report on VA facility specific Operation Enduring Freedom (OEF), Operation Iraqi Freedom (OIF), and Operation New Dawn (OND) veterans coded with potential PTSD

http://www.publichealth.va.gov/docs/epidemiology/ptsd-report-fy20 12-qtr3.pdf

Waite, L. W., \& Holder, M. D. (2003). Assessment of the emotional freedom technique: An alternative treatment for fear. Scientific Review of Mental Health Practice, 2, 20-26.

Weathers, F., Huska, J., \& Keane, T. (1991). The PTSD checklist military version (PCL-M). Boston, MA: National Center for PTSD.

Wells, S., Polglase, K., Andrews, H. B., Carrington, P., \& Baker, A. H. (2003). Evaluation of a meridian-based intervention, emotional freedom techniques (EFT), for reducing specific phobias of small animals. Journal of Clinical Psychology, 59, 943-966. doi:10.1002/jclp.10189

Zigmond, A. S., \& Snaith, R. P. (1983). The hospital anxiety and depression scale. Acta Psychiatrica Scandinavica, 67, 361-370. doi:10.1111/j.1600-0447.1983.tb09716.x 